\title{
From Hippocrates of Kos to Hashimoto and thyroid autoimmunity: A long road of discovery
}

\author{
Leonidas Duntas, ${ }^{1}$ Stefanos Geroulanos, ${ }^{2}$ Peter Laurberg ${ }^{3}$ \\ ${ }^{1}$ Unit of Endocrinology Metabolism and Diabetes, Evgenidion Hospital, University of Athens, ${ }^{2}$ International Hippocrates \\ Foundation, Kos, Greece, ${ }^{3}$ Aalborg University Hospital, Aalborg, Denmark
}

The 2012 Educational Course of the European Thyroid Association (ETA) was a truly unique event. Held $24^{\text {th }}-26^{\text {th }}$ May on the island of Kos, in the healing environment of 'the island of Hippocrates and Asclepius', it combined an educational level of excellence with the commemoration of the centennial of the description of Hashimoto thyroiditis by Hakaru Hashimoto, and the celebration of $25^{\text {th }}$ May, World Thyroid Day (WTD). The island of Kos, with its exceptionally uplifting atmosphere, was an ideal locale for a meeting focused on the recent developments in thyroid autoimmunity (AIT), while of equivalent significance was the observance of the centennial of its description by the gifted young Japanese medical scientist in 1912.

The description of Hashimoto thyroiditis in $1912^{1}$ laid the cornerstone for the merging for the first time of the fields of Thyroidology and Immunology. In addition, it was upon this groundbreaking description that a platform on which the knowledge of autoimmunity in the modern age was later to develop.

Key words: Thyroid autoimmunity, Hippocrates, Hashimoto thyroiditis

\section{Address for correspondence:}

Leonidas H. Duntas, Professor of Endocrinology, Evgenidion

Hospital, Unit of Endocrinology, Diabetes and Metabolism,

University of Athens Medical School, 11528 ,

20 Papadiamantopoulou Str., Athens, Greece,

Tel.: +30 210 6748878, e-mail: ledunt@otenet.gr

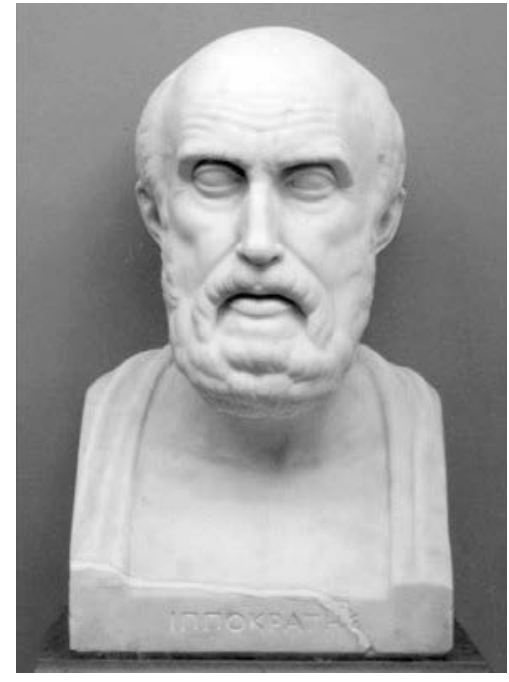

A copy of the original bust of Hippocrates at the University of Athens Medical School (sculpted by D. Koussos 1862).

There follows a brief historical overview tracing specific advances over time in western medical knowledge, as from the understanding of disease in ancient Greece of roughly 2400 years ago until today's science of Endocrinology-Immunology.

Thus, it was Hippocrates of Kos who was the first to determine that some illnesses are followed by immunity to the same disease. His description of the epidemic of mumps (parotitis epidemica) in the Greek island of Thassos, in which he uses the word anosia ( $\alpha$ vooí $\alpha$ ), is today recognized as a classic scientific observation (Epidemics I, 1,29). The word avooí $\alpha$ therein utilized is applied not only to these 


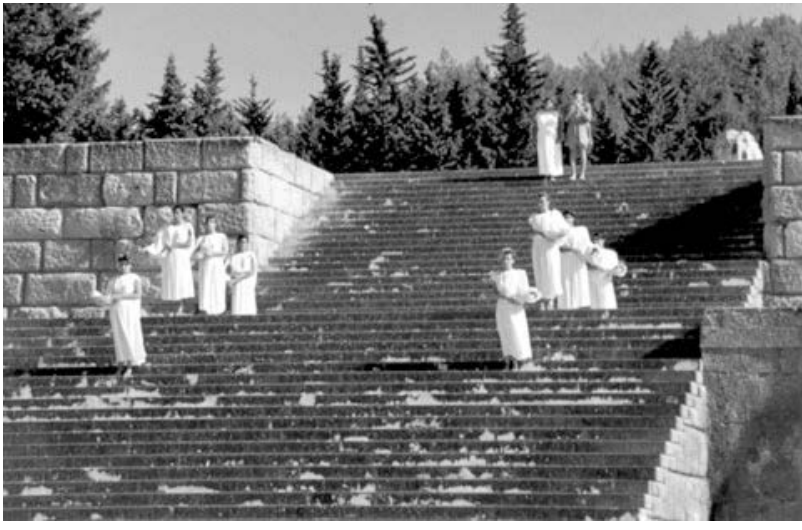

A scene of the ceremony of the re-enactment of Hippocratic Oath at the Asclepeion of Kos.

specific patients' immunity after mumps but is also cited several times in the Hippocratic Collection (Precepts 6,9-6,11; Epidemics I, 14,5; Epidemics II, 8,3 ; Letter 24,$5 ; 24,9 ; 24,24$ a.o.). Some years later Thucydides, when describing the famous plague of Athens - today identified by DNA examinations as louse-borne exanthematic typhus, due to Rickettsia prowazekii-also used the word anosos (ávoбos), stating that the disease would not a second time befall the same patient (Thucydides II, 49,6 \& 51,6).

Both Hippocrates and Thucydides applied the term anosos (formed from the word nosos, meaning disease, with the Greek privative prefix "a-") to the notion of immunity, i.e. tolerance to a specific disease, this being by distinction with "healthy". The latter is defined in Greek by the word $v \gamma \iota \eta$, stemming from the Indo-European root *su-gwi-yes literally meaning "good life", from which we have the Greek word

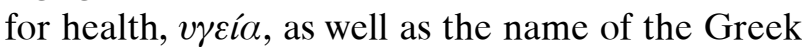
goddess, Hygeia.

The notion of autoimmunity was delineated in 1956 by the pioneering experiments conducted by N.R. Rose who immunized rabbits with thyroid extracts and described the changes in the thyroid gland, thereby anticipating Hakaru Hashimoto's description of goiter associated with thyroid lymphoid infiltration. ${ }^{2,3}$ It was in the same year, 1956, that Campbell together with Hudson, Doniach and Roitt made the notable discovery of thyroglobulin autoantibodies in Hashimoto thyroiditis and simultaneously introduced the concept of a relationship between autoimmunity and human thyroid disease. 4,5 Thyroid microsomal antibodies, produced by infiltrating lymphocytes, were discovered in 1964, these subsequently being renamed anti-TPO antibodies due to the identification of their autoantigen. ${ }^{6}$ The identification of thyroid antibodies represented a paramount achievement in elucidating the cause of thyroid autoimmunity, since the antibody titer is of great importance for the assessment of disease activity in patients with autoimmune thyroiditis.

In the meantime, it was determined that genetic factors are strongly involved in the pathogenesis of disease, with the cartography of the loci linked to AITD firmly establishing the implication of various genes. ${ }^{7,8}$ Nevertheless, after a century of investigation into this disease and despite considerable scientific advances, the precise mechanisms triggering Hashimoto thyroiditis remain largely unknown. ${ }^{9}$

The educational meeting held last May in Kos was an exceptional opportunity for a number of distinguished scientists to gather for the purposes of teaching, interacting and discussing both their results and the latest insights into a disease which still poses numerous unanswered questions.

This issue of Hormones features four of a total of six articles based on presentations delivered at the Educational Course in Kos, discussing the latest advances as regards various aspects of autoimmune thyroiditis, while they also communicate the highly congenial and animated atmosphere of the meeting inspired by the world of Medicine and the radiance of the island of Kos. The next issue of Hormones will include the remaining articles.

In their article, Yuji Hiromatsu, Satoh and Amino eloquently review the history of Hakaru Hashimoto and the discovery of thyroiditis. The tale they tell vividly illustrates the strength of mind Hakaru Hashimoto exhibited in publishing his observations, inspired by his determination to look deeper into the pathology of the thyroid gland. The authors also refer to the newly described thyroiditis, IgG4-thyroiditis, which gives evidence of being more aggressive and having higher titers of anti-TPO antibodies than the more classical form.

In a very inclusive yet concise review, Giulia Cogni 
and Luca Chiovato refer to the mechanisms inducing AITD. They initially briefly present the basic mechanisms operating in the development of thyroid autoimmunity, subsequently comprehensively describing the cellular and humoral effector mechanisms while explaining the complexities implicated in loss of tolerance to thyroid antigens and how this involves a complex interplay between genetic background and environmental factors.

Jean Louis Wemeau and colleagues vigorously stress that autoimmune thyroid diseases can be associated with other autoimmune endocrine or non-endocrine diseases. Thus, thyroid disorders are frequently expressed in adult polyendocrine syndrome type 2 , which may occur concomitantly with or secondarily to type 1 diabetes mellitus (Carpenter Syndrome), premature ovarian failure or Addison's disease (Schmidt syndrome).

Finally, Peter Laurberg and his team skillfully navigate between Scylla and Charybdis in an attempt to prevent thyroid autoimmunity by modifying iodine intake and the consumption of tobacco and alcohol. Indeed, as he describes in other studies, moderate alcohol intake may help to avoid autoimmune thyroid disease.

\section{REFERENCES}

1. Hashimoto H, 1912 Zur Kenntnis der lymphomatösen Veränderung der Schilddrüse (Struma lymphomatosa). Archiv für klinische Chirurgie 97: 219-248.

2. Rose NR, Witebsky E, 1956 Studies in organ specificity. $\mathrm{V}$ Changes in the thyroid glands of rabbits following active immunization with rabbit thyroid extracts. J Immunol 76: 417-427.

3. Campbell PN, Doniach D, Hudson RV, et al, 1956 Autoantibodies in Hashimoto's disease (lymphadenoid goitre). Lancet 271: 820-821.

4. Saravanan P, Dayan CM, 2001 Thyroid Autoantibodies. Endocrinology and Metabolism Clinics of North America 30: 315-337. doi:10.1016/S0889-8529(05)70189

5. Chardès T, Chapal N, Bresson D, et al, 2002 The human anti-thyroid peroxidase autoantibody repertoire in Graves' and Hashimoto's autoimmune thyroid diseases. Immunogenetics 54: 141-157.

6. McLachlan SM, Rapoport B, 2000 Autoimmune response to the thyroid in humans: thyroid peroxidase-the common autoantigenic. Int Rev Immunol 19: 587-618. doi:10.3109/08830180009088514.

7. Uno H, Sasazuki T, Tamai H, Matsumoto H, 1981 Two major genes, linked to 161 HLA and Gm, control susceptibility to Graves' disease. Nature 292: 768-770.

8. Tomer Y, Hasham A, Davies TF, et al, 2013 Fine mapping of loci linked to autoimmune thyroid disease identifies novel susceptibility genes. J Clin Endocrinol Metab 98: 172-173 E144-152. doi: 10.1210/jc.2012-2408.

9. Weetman AP, 2003 Autoimmune Thyroid Disease: propagation and progression. Eur J Endocrinol 148: 1-9. 